\title{
Death at the airway epithelium in asthma
}

\author{
Cell Research (2013) 23:588-589. doi:10.1038/cr.2013.26; published online 19 February 2013
}

\begin{abstract}
Allergic asthma is an inflammatory disease of the airways in which epithelial cells, dendritic cells and innate lymphoid cells are increasingly implicated. Recent findings suggest that apoptotic cells are phagocytosed by airway epithelial cells in a Rac1dependent manner and this leads to dampening of innate and adaptive immunity to allergens.
\end{abstract}

Allergic asthma is a chronic inflammatory disease of the airways, characterized by the infiltration of eosinophils, basophils and mast cells into the airway submucosa and airway smooth muscle. The immune response to inhaled allergens is characterized by an aberrant Th2 lymphocyte response that causes the features of asthma, through the production of IL-4, IL-5 and IL-13. Type 2 innate lymphocytes (ILC2s) are also known as nuocytes or natural helper cells and produce copious amounts of IL-5, IL-9 and IL-13. ILC2s belong to an expanding family of innate lymphocytes that do not express a B cell or T cell receptor, and include NK cells, NKp $46^{+}$IL-22 producing ILCs and lymphoid tissue inducer cells (LTi) [1]. In mice, ILC2s derive from committed T1/ST2 ${ }^{+}$pre-ILC2s that develop from common lymphoid progenitors under the influence of IL-33 and/or IL-25, and depend on the transcription factors ROR $\alpha$ and GATA3. These cells are recruited to the lungs of allergen-exposed mice, and partially control eosinophilic influx and bronchial hyperreactivity by producing IL-5 and IL-13, independently of adaptive immunity.

Recent insights have put the airway epithelium in the spotlight of the process of allergic sensitization through their potential to activate dendritic cells (DCs) (reviewed in [2]). DCs are crucial inducers of Th2 immunity to allergens [3]. As epithelial cells (ECs) represent the first line of defence to inhaled allergens and also express Toll-like receptors (TLRs) and other pattern recognition receptors (PRRs), they have the ability to sense the same stimuli as innate immune cells. Triggering of PRRs initiates NF- $\kappa \mathrm{B}$ activation and leads to the release of pro-Th2 cytokines such as thymic stromal lymphopoietin (TSLP), GM-CSF, IL-1 $\alpha$, IL-25 and IL-33 [4]. Recently, IL-33 has been shown to act upstream of the type- 2 effector cytokine cascade, by stimulation of DCs, ILC2s and adaptive immune cells. Asthmatic subjects express higher levels of IL-33 in mucosal biopsies and serum compared with healthy controls [5]. Recent GWAS studies on the genetics of asthma have also identified single nucleotide polymorphisms (SNPs) in the gene coding for the IL-33 receptor IL-1RL1 as well as in the IL-33 locus itself [6].

Despite the clear effects of IL-33 on promoting ILC2 development and DC activation, less is known about IL33 release from stromal and immune cells, and whether IL-33 release results from cell death mechanisms or active secretion. The confusion stems from the fact that IL-33 does not contain a signaling peptide for secretion through the endoplasmic reticulum and Golgi pathway. IL-33 is possibly released in a passive manner as a result of necrotic cell death acting as an alarmin. During necrosis IL-33 remains in its active form, whereas under conditions of apoptotic cell death the executor caspases- 3 and -7 cleave IL-33 into an inactive form [7]. In a house dust mite (HDM)-driven murine model of asthma, the epithelial repair factor Trefoil factor 2 (TFF-2) was shown to induce IL-33 production in airway epithelia, alveolar macrophages and $\mathrm{FceRI} \mathrm{I}^{+}$inflammatory DCs and thus contribute to induction of Th2 immunity [8]. Other alarmins like HM-GB1, uric acid and ATP that are typically released in response to necrotic cell death, also seem to be released at an increased rate in asthmatic airways, but again it is unclear whether they are released from dying cells or by an active process [9].

A recent report in Nature adds a further level of complexity to the function of airway ECs and cell death [10]. The authors demonstrate that apoptotic ECs are engulfed by viable bronchial ECs in a process requiring the small GTPase Rac1, classically involved in the engulfment of large extracellular material by phagocytes and DCs. Deficiency of Rac1 selectively in mouse bronchial ECs led to defective uptake of injected apoptotic ECs in vitro and in vivo. Engulfment of apoptotic cells by ECs leads to the production of IL-10 and TGF $\beta$, thus suppressing the activation of immune cells (Figure 1). Subsequently, the authors show that administration of endotoxin-low ovalbumin or HDM to mice lacking Rac1 in bronchial ECs leads to increased development of Th2 immunity and the salient features of asthma, whereas this is not the case in mice expressing wild-type (WT) Rac1. These findings are at odds with several other studies in which administration of HDM to the airways of WT mice was able to induce Th2 immunity to HDM and to asthma. One likely explanation is 
that the HDM extracts used in the Juncadella paper was very low in endotoxin content. It is known that endotoxin is necessary to provide epithelial activation for innate pro-Th2 cytokine production [11]. When bronchial ECs lacked Rac1, there was an increase in IL-33 production, known to activate DCs and ILC2s, upon low-dose HDM exposure. This low-dose IL-33 might have pulled the trigger on innate immune recognition of the endotoxin-low HDM.

This paper raises the interesting concept that dying ECs dampen the threshold for allergen recognition. Several questions remain however. First, what is the source of dying ECs? It is known that dead ECs are found in the airways of patients (so called Creola bodies), but it remains to be shown whether these cells died by necrosis, apoptosis or necroptosis (programmed necrosis). In our own experience, there is little evidence for cell death in the airways of mice exposed to HDM allergen, so this issue will have to be resolved in the future. Second, IL-33 release is very complicated and itself regulated by caspases and elastastes, other cytokines like IL- $1 \alpha$ as well as the TFF-2. It is uncertain at this stage how recognition and internalization of dead cells interferes with these pathways of IL-33 secretion. Although the paper by Juncadella et al. shows an increase of ILC2s in mice lacking Rac1 in ECs, the precise role of ILC2s in asthma is incompletely understood. Another piece of the puzzle is the cellular mechanism by which IL10 and TGF $\beta$ are produced in response to apoptotic cell recognition and how IL-10 and TGF $\beta$ subsequently suppress allergy development. IL-10 and TGF $\beta$ can suppress the function of DCs, but could also induce the production of regulatory $\mathrm{T}$ cells. Finally, it will be very interesting to study whether and how this pathway is also operative in humans, and whether individuals prone

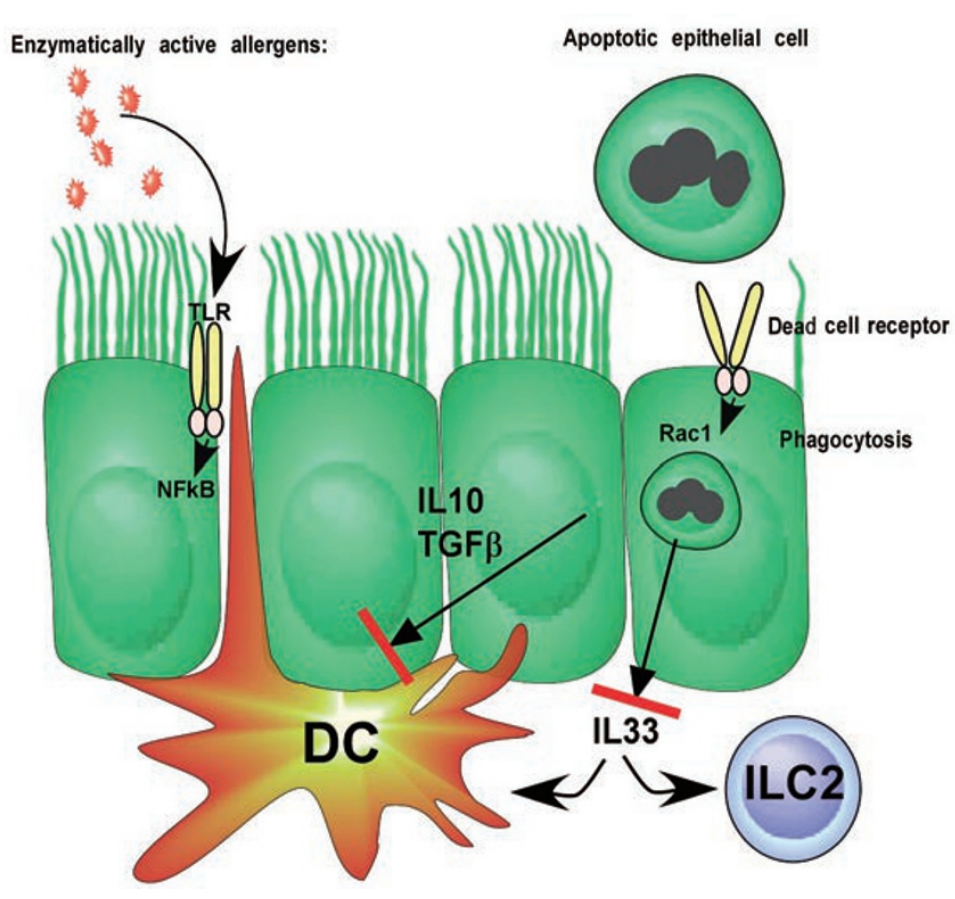

Figure 1 Effect of uptake of apoptotic cells on process of allergic sensitization. Apoptotic cells are endocytosed via an unknown dead cell receptor in a process requiring the small GTPase Rac1. Engulfment of apoptotic cells leads to secretion of IL-10 and TGF $\beta$ that most likely suppresses the functions of DCs. Additionally there is a block of IL-33 production, suppressing the activation of DCs and ILC2s. DCs are crucial in mounting Th2 immunity to allergens, whereas ILC2s are emerging as effector cells in allergy by secretion of IL-5 and IL-13.

to developing allergy and asthma have polymorphisms in the genes controlling the uptake of apoptotic cells by ECs, or whether ongoing asthmatic airway inflammation interferes with the recognition of dead cells in the lungs. Finding therapeutics or intervention strategies that exploit this anti-inflammatory pathway of ECs could be valuable tools in the fight against asthma and allergies.

\footnotetext{
Bart N Lambrecht ${ }^{1,2,3}$, Hamida Hammad ${ }^{1,2}$

${ }^{1}$ Department for Molecular Biomedical Research, VIB, Gent, Belgium; ${ }^{2}$ Department of Pulmonary Medicine, Ghent University, Ghent, Belgium; ${ }^{3}$ Department of Pulmonary Medicine, Erasmus MC, Rotterdam, The Netherlands Correspondence: Bart N Lambrecht E-mail: bart.lambrecht@ugent.be
}

\section{References}

1 Walker JA, Barlow JL, McKenzie AN. Nat Rev Immunol 2013; 13:75-87.

2 Lambrecht BN, Hammad H. Nat Med 2012; 18:684-692.

3 Plantinga M, Guilliams M, Vanheerswynghels M, et al. Immunity 2013; 38:322-335.

4 Willart MA, Deswarte K, Pouliot P, et al. J Exp Med 2012; 209:1505-1517.

5 Kurowska-Stolarska M, Stolarski B, Kewin $\mathrm{P}$, et al. J Immunol 2009; 183:6469-6477.

6 Moffatt MF, Gut IG, Demenais F, et al. $N$ Engl J Med 2010; 363:1211-1221.

7 Lüthi AU, Cullen SP, McNeela EA, et al. Immunity 2009; 31:84-98.

8 Wills-Karp M, Rani R, Dienger K, et al. J Exp Med 2012; 209:607-622.

9 Kool M, Willart MA, van Nimwegen M, et al. Immunity 2011; 34:527-540.

10 Juncadella IJ, Kadl A, Sharma AK, et al. Nature 2013; 493:547-551.

11 Hammad H, Chieppa M, Perros F, et al. Nat Med 2009; 15:410-416. 